\title{
Robust evidence for the stabilization of the premartensite phase in Ni-Mn-In magnetic shape memory alloys by chemical pressure
}

\author{
Anupam K. Singh $\odot,{ }^{1}$ Sanjay Singh $\odot,{ }^{1, *}$ B. Dutta $\odot,{ }^{2}$ K. K. Dubey, ${ }^{1}$ Boby Joseph $\odot,{ }^{3}$ R. Rawat $\odot,{ }^{4}$ and Dhananjai Pandey ${ }^{1}$ \\ ${ }^{1}$ School of Materials Science and Technology, Indian Institute of Technology (Banaras Hindu University), Varanasi-221005, India \\ ${ }^{2}$ Department of Materials Science and Engineering, Faculty of Mechanical, Maritime and Materials Engineering, \\ Delft University of Technology, Mekelweg 2, 2628 CD Delft, The Netherlands \\ ${ }^{3}$ Elettra-Sincrotrone Trieste, Strada Statale 14, Km 163.5 in Area Science Park, Basovizza 34149, Italy \\ ${ }^{4}$ UGC-DAE Consortium for Scientific Research, University Campus, Khandwa Road, Indore 452001, India
}

(Received 20 June 2021; revised 30 September 2021; accepted 22 October 2021; published 30 November 2021)

\begin{abstract}
The thermodynamic stability of the premartensite (PM) phase has been a topic of extensive investigation in shape memory alloys as it affects the main martensite phase transition and the related physical properties. In general, the PM phase is stable over a rather narrow temperature-composition range. We present here evidence for chemical pressure induced suppression of the main martensite transition and stabilization of the PM phase over a very wide temperature range from 300 to $\sim 5 \mathrm{~K}$ in a magnetic shape memory alloy (MSMA), $\mathrm{Ni}_{50} \mathrm{Mn}_{34} \mathrm{In}_{16}$, using magnetic susceptibility, synchrotron x-ray powder diffraction (SXRPD) studies, and first-principles calculations. The ac-susceptibility studies show a highly skewed and smeared peak around $300 \mathrm{~K}$ without any further transition up to the lowest temperature of our measurement $(5 \mathrm{~K})$ for $\sim 5 \% \mathrm{Al}$ substitution. The temperature evolution of the SXRPD patterns confirms the appearance of the PM phase related satellite peaks at $T \leqslant 300 \mathrm{~K}$ without any splitting of the main austenite (220) peak showing preserved cubic symmetry. This is in marked contrast to the temperature evolution of the SXRPD patterns of the martensite phase of the $\mathrm{Al}$ free as well as $\sim 3 \% \mathrm{Al}$ substituted compositions where the austenite (220) peak shows a clear splitting due to Bain distortion signalling symmetry breaking transition. Our theoretical calculations support the experimental findings and reveal that the substitution at the In site by a smaller size atom, like Al, can stabilize the PM phase with preserved cubic symmetry. Our results demonstrate that Al-substituted Ni-Mn-In MSMAs provide an ideal platform for investigating the physics of various phenomena related to the PM state.
\end{abstract}

DOI: 10.1103/PhysRevMaterials.5.113607

\section{INTRODUCTION}

Large shape change under the influence of external stress and its recovery on annealing above a characteristic temperature is the key property of a class of functional materials named shape memory alloys (SMAs) [1-4]. The origin of the large recoverable shape change (strain) in SMAs is intimately linked with a diffusionless martensite phase transition in which a higher symmetry cubic austenite phase transforms to a lower symmetry martensite phase with tetragonal/orthorhombic/monoclinic Bain distortion [5-7]. The martensite transition in SMAs is a reversible transition, unlike in the steels where it is irreversible [8]. An interesting precursor or premartensite (PM) transition has been reported to precede the martensite phase transition in some of the SMAs $[3,9,10]$. This phenomenon and its role on the structure-property correlations in various SMAs has since been extensively investigated [3,10-15]. The precursor PM phase occurs at an intermediate temperature range between high temperature austenite and low temperature martensite phase with preserved cubic symmetry of the austenite phase [3,10-15].

In recent years, the appearance of the PM phase has received considerable attention in another class of SMAs,

\footnotetext{
*ssingh.mst@iitbhu.ac.in
}

known as magnetic shape memory alloys (MSMAs) [16-24]. The advantage of the MSMAs over the SMAs is that the magnetic field-induced shape change is accompanied with much larger strain (MFIS) which can be recovered within the martensite phase itself without any annealing above the transition temperature $[25,26]$. Also, the response time associated with the occurrence of the magnetic field-induced strain and its recovery is much faster than that in the conventional SMAs [25] opening the possibility for the development of a new class of multifunctional sensors and actuators based on application/removal of magnetic field without any temperature variation [25]. Besides the large MFIS [26-28], the MSMAs have received tremendous interest in recent past due to the observation of several other exciting phenomena like large magnetocaloric effect [29-33], giant magnetoresistance [34-37], anomalous thermal properties [38,39], exchange bias effect [40,41], spin glass [42], giant Hall effect [43], and anomalous Nernst effect [44], all of which have great potential for technological applications. The study of the precursor PM phase in the MSMAs is of crucial importance in relation to several exotic phenomena like strain glasses [45] and skyrmions [24] in MSMAs.

Among the various MSMAs, the crystal structure, phase stability, and the magnetization behavior of the PM phase have been extensively investigated in the Ni-Mn-Ga system $[16,18,21,22,46,47]$. For example, the temperature 
dependence of the dc magnetization in some of these alloys shows a small dip/peak at the PM transition [18,19,22] temperature at which it changes by $\sim 2 \%$ as compared to that of the austenite phase [22,23]. In contrast, the martensite phase transition is accompanied by a huge change $(>40 \%)$ in magnetization [22,23] due to large Bain distortion and therefore much higher magnetocrystalline anisotropy of the martensite phase [48]. The PM phase of the near stoichiometric $\mathrm{Ni}_{2} \mathrm{MnGa}$ is characterized by the appearance of very weak intensity satellite peaks in the diffraction pattern even as the cubic austenite phase peaks remain almost unaffected due to the absence of any discernible Bain distortion [21,22]. As a result, the PM phase has been regarded as a micromodulated precursor state with preserved cubic symmetry [18]. This is in contrast to the martensite phase in which, besides the appearance of the new satellite peaks, cubic austenite peaks also split due to significant Bain distortion signaling a symmetry breaking transition [21,22]. Recently, we presented evidence for the thermodynamic stability of both the PM and the martensite phases using a detailed temperature dependent synchrotron X-ray powder diffraction (SXRPD) study on $\mathrm{Ni}_{2} \mathrm{MnGa}$ [22].

The intermediate PM phase has been a subject matter of investigation in a few Ga-free Ni-Mn- $X(X=\mathrm{Sn}$ and In) MSMAs $[23,24,49]$ also. For example, in Co-doped Ni$\mathrm{Mn}-\mathrm{Sn}$ alloys, obtained after pressure annealing [50,51], the appearance of the precursor PM phase has been attributed to enhanced magnetoelastic coupling [18,19,23,52]. Chemical pressure, generated by substitution with smaller size atoms, like $\mathrm{Al}$ at the In site in the Ni-Mn-In alloy composition, has also been reported to stabilize the PM phase over a modest temperature range of about $40 \mathrm{~K}$ [23]. The stabilization of the PM phase has also been reported in the conventional shape memory alloys like NiTi after partial substitution of Ni with Fe [3] and in $\mathrm{Ni}_{x} \mathrm{Al}_{100-x}$ for $x<60$ [10,53], by chemical pressure tuning.

The foregoing results highlight the importance of chemical pressure-tuning of the transition temperature leading to the stabilization of the precursor PM phase and destabilization of the martensite phase. However, all these studies are mainly based on bulk magnetic measurements without any temperature dependent structural studies. Since the laboratory source x-ray power diffraction data often fails to capture the signatures of the PM phase [54], any structural confirmation of the chemical pressure-tuned PM phase in MSMAs requires high-resolution SXRPD data, which not only reveal the presence of characteristic weak satellite peaks due to its high signal to noise ratio but also its ability to capture the Bain distortion, if present, and hence the signature of the symmetry breaking transition, unambiguously due to the high peak to peak resolution $[22,46,55]$. In the present work, we have carried out a comprehensive study on the effect of the chemical pressure generated by $\mathrm{Al}$ substitution in place of In in $\mathrm{Ni}_{50} \mathrm{Mn}_{34} \mathrm{In}_{16}$ MSMA using bulk magnetic measurements, temperature dependent high-resolution SXRPD studies, and first-principles calculations. Our magnetic susceptibility studies on these alloys suggest that Al substitution in place of In destabilizes the martensite phase and stabilizes the PM phase over a wider temperature range. Our temperature dependent high resolution SXRPD studies on these alloys reveal Bain distortion in the martensite phase and its absence in the PM phase. The bulk magnetic and structural studies show that $\mathrm{Al}$ free $\mathrm{Ni}_{50} \mathrm{Mn}_{35} \mathrm{In}_{15}$ MSMA exhibits only the martensite phase of $3 M$ type in the monoclinic space group $P 2 / m$ with significant Bain distortion [56]. However, in the $\sim 3 \% \mathrm{Al}$ substituted $\mathrm{Ni}_{50} \mathrm{Mn}_{34} \mathrm{In}_{15.5} \mathrm{Al}_{0.5}$ alloy, a $\mathrm{PM}$ phase, stable over a narrow temperature window of $\sim 10 \mathrm{~K}$ below the ferromagnetic $T_{C} \sim$ $317 \mathrm{~K}$, precedes the appearance of the martensite phase at lower temperatures. The PM to martensite phase transition is shown to be an isostructural phase transition as both the phases are of $3 M$ type in the monoclinic $P 2 / m$ space group and differ only in terms of the absence or otherwise of the Bain distortion. More significantly, we show that on increasing the Al content to $\sim 5 \%$ (i.e., $\mathrm{Ni}_{50} \mathrm{Mn}_{34} \mathrm{In}_{15.2} \mathrm{Al}_{0.8}$ ), only the PM phase, without any Bain distortion, occurs over the entire temperature range (300-5 K) below the PM transition temperature $T_{\mathrm{PM}} \sim 300 \mathrm{~K}$ without any signature of the martensite phase transition either in the magnetic or the structural studies. We also show that the $T_{\mathrm{PM}}$ decreases with increasing magnetic field and the satellite peaks of the PM phase disappear in the presence of external magnetic field confirming the magnetoelastic coupling in this alloy composition. Using first-principles calculations, we further investigate the energetics of alloys which provide atomistic insight into the stabilization of PM phase in Al substituted Ni-Mn-In MSMA. Our results demonstrate that Al substituted Ni-Mn-In MSMAs provide an ideal platform for investigating the physics of PM phase related phenomena in MSMAs.

\section{RESULTS AND DISCUSSION}

The details of sample preparation, measurements, and analysis are given in the Supplemental Material [57]; also see Refs. [58-68]. Al-free $\mathrm{Ni}_{50} \mathrm{Mn}_{35} \mathrm{In}_{15}$ (or $\mathrm{Ni}_{2} \mathrm{Mn}_{1.4} \mathrm{In}_{0.6}$ ) MSMA [56] exhibits paramagnetic to ferromagnetic (FM) transition with $T_{C} \sim 315 \mathrm{~K}$, a first order austenite to martensite transition in the $\mathrm{FM}$ phase at $T_{M} \sim 295 \mathrm{~K}$ with a characteristic thermal hysteresis in the temperature dependent magnetization $M(T)$ plots for the field cooled (FC) and field cooled warming (FCW) protocols and another transition at $T_{C}^{M} \sim 150 \mathrm{~K}$, commonly attributed to competing FM and antiferromagnetic (AFM) interactions [69,70], with bifurcation of the zero field cooled warming (ZFCW) and FC $M(T)$ plots in the dc magnetization studies [56]. While the FM $T_{C}$ of the base alloy $\left(\mathrm{Ni}_{50} \mathrm{Mn}_{35} \mathrm{In}_{15}\right)$ is known to be nearly unaffected by $\mathrm{Al}$ substitution $[23,36]$, the nature of transitions below $T_{C}$ changes rather drastically as a function of $\mathrm{Al}$ content. This can be seen from a comparison of magnetization data of the base alloy in Ref. [56] with those given in Figs. 1(a) and 1(b). For the $\sim 3 \%$ Al substituted composition $\left(\mathrm{Ni}_{50} \mathrm{Mn}_{34} \mathrm{In}_{15.5} \mathrm{Al}_{0.5}\right)$ one observes, two peaks at $T_{\mathrm{PM}} \sim 311 \mathrm{~K}$ and $T_{M} \sim 300 \mathrm{~K}$ in real part of ac-susceptibility $\left[\chi^{\prime}(T)\right]$ plot shown in the inset of Fig. 1(a) corresponding to the PM and martensite transitions, respectively. Both the transitions exhibit characteristic thermal hysteresis in $\chi^{\prime}(T)$ plots for the FC and FCW protocols, shown in the main figure [Fig. 1(a)], suggesting their first order character. The nature of the two transitions shown in Fig. 1(a) are in broad agreement with those reported in a previous study $[23,49]$, even though the transition temperatures and behavior of $\chi^{\prime}(T)$ are somewhat different, possibly due to a small fluctuations in the alloy composition [70-74]. 


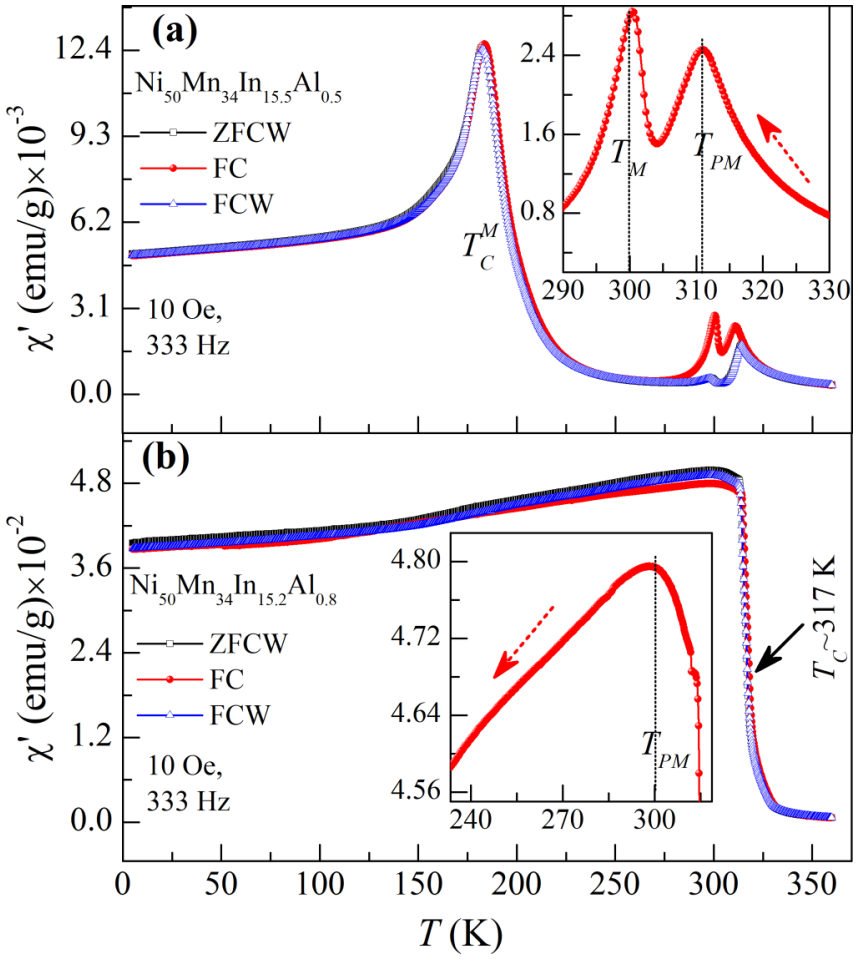

FIG. 1. The temperature dependent real part of ac-susceptibility for (a) $\mathrm{Ni}_{50} \mathrm{Mn}_{34} \mathrm{In}_{15.5} \mathrm{Al}_{0.5}$ and (b) $\mathrm{Ni}_{50} \mathrm{Mn}_{34} \mathrm{In}_{15.2} \mathrm{Al}_{0.8}$ MSMAs. The insets are enlarged view around $300 \mathrm{~K}$ for the field cooled protocol. $T_{M}, T_{\mathrm{PM}}, T_{C}^{M}$, and $T_{C}$ represent the martensite transition temperature, premartensite transition temperature, Curie temperature of the martensite phase, and Curie temperature of the austenite phase, respectively. ZFCW, FC, and FCW correspond to measurements performed during warming on the zero field cooled sample, during field cooling, and during warming on the field cooled sample, respectively.

We now proceed to correlate the two anomalies in $\chi^{\prime}(T)$ of $\mathrm{Ni}_{50} \mathrm{Mn}_{34} \mathrm{In}_{15.5} \mathrm{Al}_{0.5}$ with PM and martensite phase transitions using structural studies. Figures 2(a)-2(c) depict the SXRPD patterns of $\mathrm{Ni}_{50} \mathrm{Mn}_{34} \mathrm{In}_{15.5} \mathrm{Al}_{0.5}$ recorded at $400 \mathrm{~K}\left(>T_{C}\right)$, $310 \mathrm{~K}$, and $110 \mathrm{~K}$, respectively. The emergence of new peaks in these SXRPD patterns at 310 and $110 \mathrm{~K}$ reveal structural changes related with the PM and martensite transitions, respectively. All the peaks in Fig. 2(a) can be indexed with the austenite cubic structure in the $F m \overline{3} m$ space group, as confirmed by LeBail refinement, the details of which are given in Fig. S1(a) (see the Supplemental Material [57]). The cubic lattice parameter obtained after the refinement is found to be $a=6.01009(6) \AA$ A. Further, the presence of the (111) and (200) Bragg reflections [see inset (i) of Fig. S1(a) of the Supplemental Material [57]] confirms the $L 2_{1}$ ordering above the FM $T_{C} \sim 317 \mathrm{~K}$. At $T \sim 310 \mathrm{~K}\left(<T_{\mathrm{PM}}\right)$, new satellite peaks with very low intensities appear, as can be seen from the inset (i) of Fig. 2(b), which gives the SXRPD plot on a magnified scale for a limited $2 \theta$ range. All the peaks, including the satellite peaks, in this pattern are well accounted for by a $3 M$ modulated monoclinic structure in the $P 2 / \mathrm{m}$ space group with $a=4.3869$ (7) $\AA, b=5.6866(1) \AA, c=13.0028(2) \AA$, and $\beta=93.695(3)^{\circ}$ as can be seen from Fig. S1(b) (see the Supplemental Material [57]) which gives the results of LeBail refinement for the SXRPD pattern at $\sim 310 \mathrm{~K}$. At this temper-

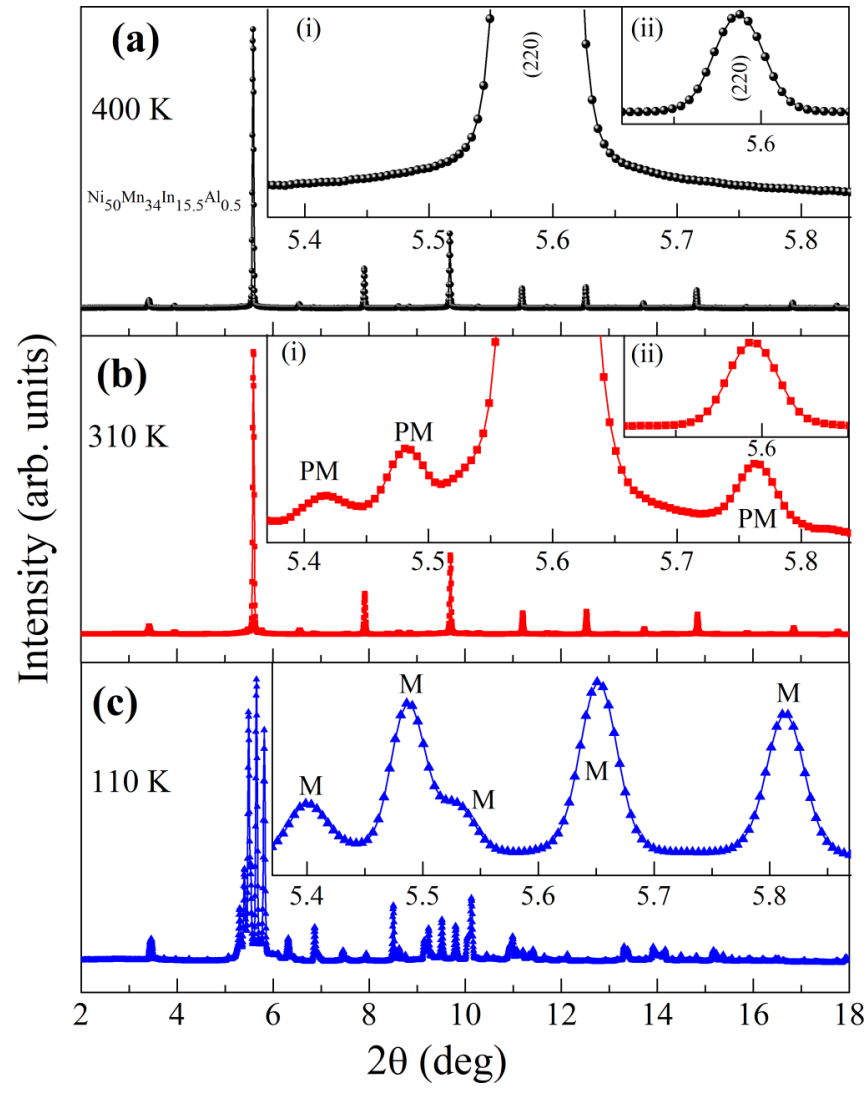

FIG. 2. Typical SXRPD patterns of $\mathrm{Ni}_{50} \mathrm{Mn}_{34} \mathrm{In}_{15.5} \mathrm{Al}_{0.5}$ MSMA in the (a) austenite, (b) premartensite, and (c) martensite phases. An enlarged view around the most intense (220) Bragg peak for the austenite and the premartensite (PM) phases, given in inset (i) of (a) and (b), respectively, reveal the appearance of the satellite peaks [indicated by "PM" in the inset (i) of (b)] due to $3 M$ like modulation in the PM phase. Untruncated view of the (220) cubic peak for the austenite and PM phases, given in inset (ii) of (a) and (b), respectively, reveal the absence of Bain distortion in the PM phase. The inset of (c) depicts the splitting of the most intense (220) cubic peak and appearance of the satellite peaks due to Bain distortion and $3 M$ like modulation of the martensite $(\mathrm{M})$ phase. The peaks related to the martensite phase are marked as "M" in the inset of (c).

ature, the "cubic" peaks do not show any splitting [see inset (ii) of Fig. 2(b)] confirming the appearance of the PM phase below $T_{\mathrm{PM}}$ with preserved cubic symmetry without any discernible Bain distortion, similar to the $\mathrm{PM}$ phase of $\mathrm{Ni}_{2} \mathrm{MnGa}$ [46,75]. On lowering the temperature further below $T_{M}$, the intensity of the existing satellite peaks increases considerably while the cubic peaks split into multiple peaks [see the inset of Fig. 2(c)] due to significant Bain distortion, as expected for the martensite phase [56]. LeBail refinement for the $110 \mathrm{~K}$ SXRPD pattern confirms that all the peaks in the martensite phase are also accounted for using the monoclinic $P 2 / \mathrm{m}$ space group [see Fig. S1(c) of the Supplemental Material [57]]. We also verified the stability of the martensite phase from $300 \mathrm{~K}$ down to $13 \mathrm{~K}$ using $\mathrm{x}$-ray powder diffraction data obtained from an $18-\mathrm{kW} \mathrm{Cu}$ rotating anode based high resolution diffractometer fitted with a curved graphite crystal monochromator in the diffraction beam and a close-cycle He refrigerator-based low temperature attachment. The corre- 


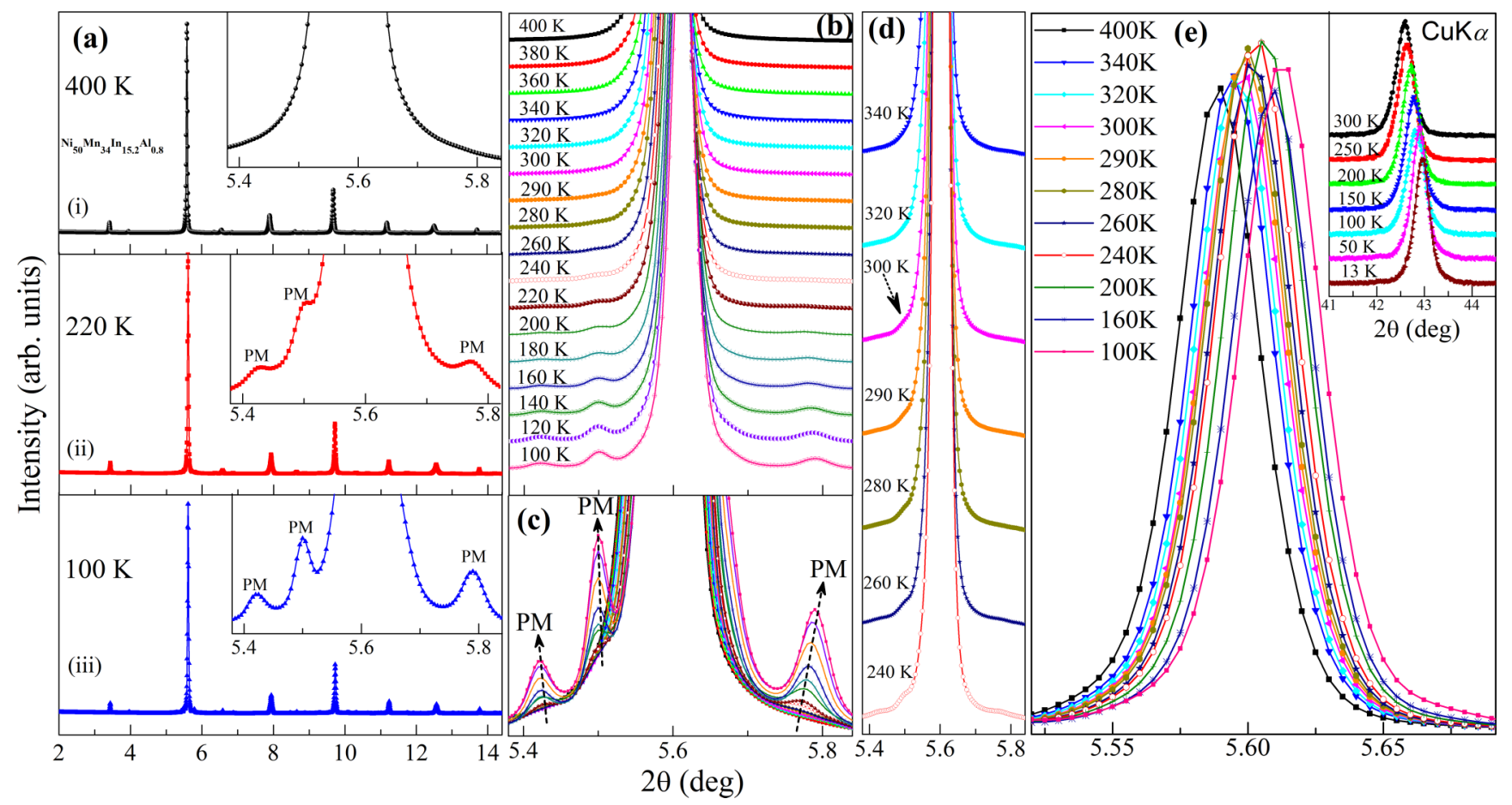

FIG. 3. The SXRPD patterns of $\mathrm{Ni}_{50} \mathrm{Mn}_{34} \mathrm{In}_{15.2} \mathrm{Al}_{0.8}$ are shown in (a) at (i) $400 \mathrm{~K}$, (ii) $220 \mathrm{~K}$, and (iii) $100 \mathrm{~K}$. The insets show an enlarged view around the most intense Bragg peak to reveal the satellite peaks of the premartensite (PM) phase. Enlarged view around the most intense cubic peak (220) at various temperature in the range 400-100 K are given in (b) and (c). The arrows in (c) indicate the temperature dependent shifts of the PM satellite peak positions. Note the gradual sharpening of the satellite peaks in (c) on lowering the temperature. (d) An enlarged view around the most intense (220) cubic peak at selected temperatures reveal the appearance of the most intense satellite peak of the PM phase at $T \sim 300 \mathrm{~K}$, indicated by an arrow. (e) Untruncated SXRPD profiles of the (220) cubic Bragg peak is depicted in the 400-100-K range while its inset depicts the XRD profiles in the 300-13-K range recorded on a rotating anode-based diffractometer.

sponding $\mathrm{x}$-ray diffraction (XRD) patterns in the $20-100^{\circ} 2 \theta$ range are depicted in Fig. S2 (see the Supplemental Material [57]). Thus, the SXRPD studies on the $\mathrm{Ni}_{50} \mathrm{Mn}_{34} \mathrm{In}_{15.5} \mathrm{Al}_{0.5}$ alloy above $300 \mathrm{~K}$ reveal cubic austenite to monoclinic (space group $P 2 / m$ ) PM phase transition at $T_{\mathrm{PM}}$ without any Bain distortion, as revealed by the absence of any splitting of the cubic peaks while the SXRPD and rotating anode based laboratory source data reveal an isostructural PM to martensite phase transition with significant Bain distortion, as revealed by the splitting of the cubic peaks in the martensite phase.

On increasing the $\mathrm{Al}$ content from $\sim 3 \%$ to $\sim 5 \%$ (i.e., $\mathrm{Ni}_{50} \mathrm{Mn}_{34} \mathrm{In}_{15.2} \mathrm{Al}_{0.8}$ MSMA), the phase transition behavior changes drastically. Figure 1(b) shows temperature dependence of the $\chi^{\prime}(T)$ for $\sim 5 \%$ Al substituted alloy, $\mathrm{Ni}_{50} \mathrm{Mn}_{34} \mathrm{In}_{15.2} \mathrm{Al}_{0.8}$. For the $\mathrm{FC}$ protocol, the paramagnetic to FM transition occurs at $T_{C} \sim 317 \mathrm{~K}$ with a sharp increase in the $\chi^{\prime}(T)$, comparable to the $T_{C}$ of the base alloy as can be seen from a comparison of Fig. 1(b) with the temperature dependence of dc magnetization of the base alloy in Ref. [56]. On decreasing the temperature further, the rate of increase of $\chi^{\prime}(T)$ decreases before peaking at the PM transition temperature $T_{\mathrm{PM}} \sim 300 \mathrm{~K}$, as can be seen from the inset of Fig. 1(b). This peak is highly skewed and smeared out on the lower temperature side down to $\sim 5 \mathrm{~K}$. The gradually decreasing trend of $\chi^{\prime}(T)$ below $T_{\mathrm{PM}}$ is in marked contrast to its sharp drop in dc magnetization of the base alloy [56] which is usually attributed to a very large magnetocrystalline anisotropy of the martensite phase [48]. More significantly, there is no signature of the second anomaly, seen in Fig. 1(a) for $\mathrm{Ni}_{50} \mathrm{Mn}_{34} \mathrm{In}_{15.5} \mathrm{Al}_{0.5}$, corresponding to the martensite transition in $\mathrm{Ni}_{50} \mathrm{Mn}_{34} \mathrm{In}_{15.2} \mathrm{Al}_{0.8}$.

The absence of martensite transition and stabilization of the PM phase indicated by the $\chi^{\prime}(T)$ plot in Fig. 1(b) was confirmed by SXRPD studies at selected temperatures in the $400-100-\mathrm{K}$ range and laboratory source XRD patterns at several temperatures in the 300-13-K range. Figure 3(a) compares the SXRPD patterns of $\mathrm{Ni}_{50} \mathrm{Mn}_{34} \mathrm{In}_{15.2} \mathrm{Al}_{0.8}$ alloy at 400,220 , and $100 \mathrm{~K}$. The insets in panels (i)-(iii) depict a magnified view of the profiles in the $2 \theta$ range $5.38-5.84^{\circ}$ around the most intense Bragg peak. The inset of (ii) reveals the presence of satellite peaks at $220 \mathrm{~K}$ whose intensity increases on lowering the temperature to $100 \mathrm{~K}$ [see inset of (iii)]. These satellite peaks are absent at $400 \mathrm{~K}$, as can be seen in the inset of (i). The evolution of the SXRPD profiles as a function of temperature is shown in Figs. 3(b) and $3(\mathrm{c})$ at close temperature interval using a magnified (vertically zoomed) view of the intensity profile around the most intense cubic (220) peak. The most intense satellite appears around $300 \mathrm{~K}$ as shown with an arrow in Fig. 3(d), in agreement with the transition temperature $T_{\mathrm{PM}}$ corresponding to the austenite to PM transition in the $\chi^{\prime}(T)$ plot shown in Fig. 1(b). The intensity of the three prominent satellite peaks around the (220) cubic peak keeps growing below $300 \mathrm{~K}$, as can be seen from Figs. 3(b) and 3(c). Further, the FWHM of the satellite peaks decreases [see Fig. 3(c)] with decreasing temperature suggesting that the correlation length or the 

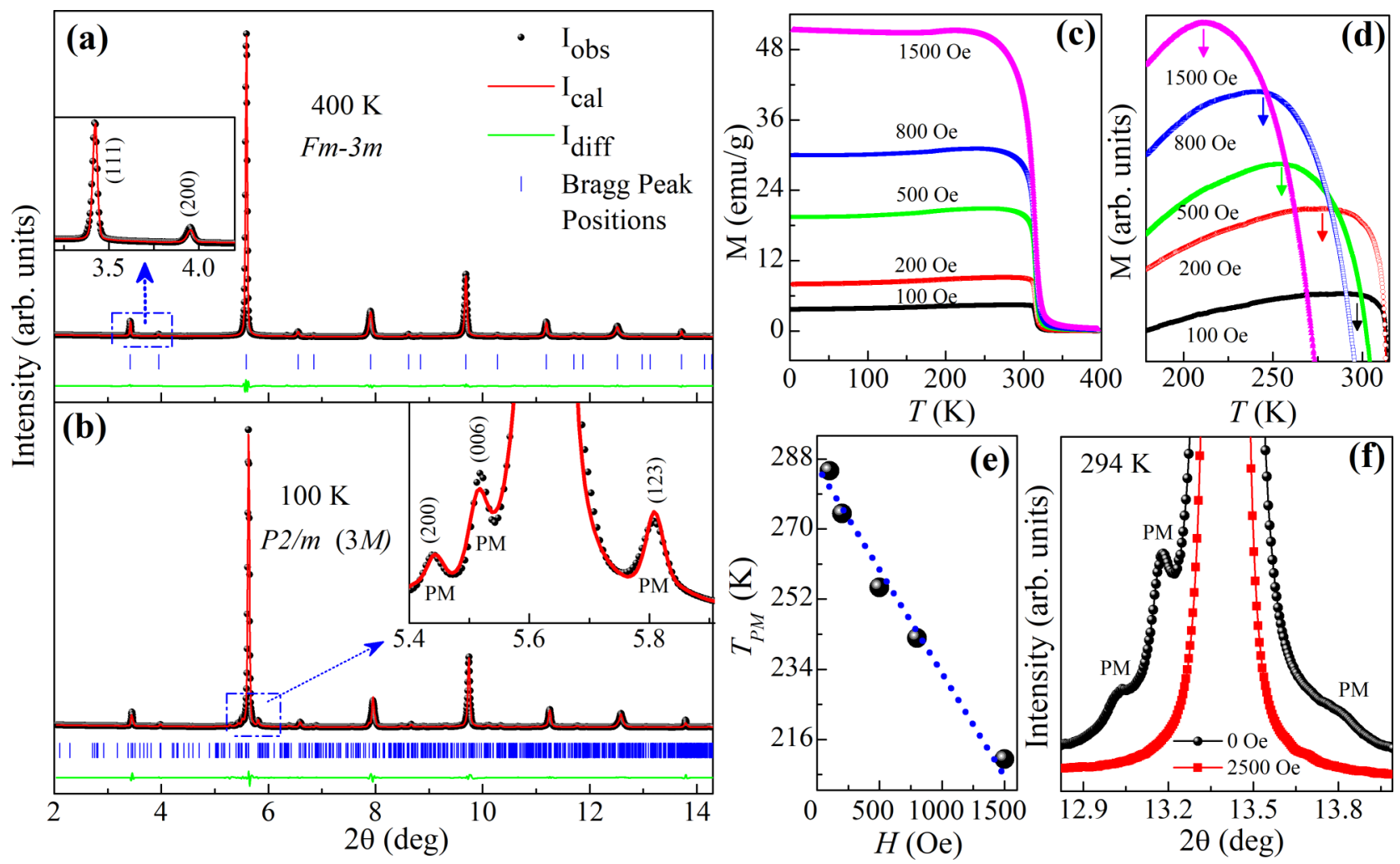

FIG. 4. The observed (dark black dots), calculated (continuous red line), and difference patterns (continuous green line) obtained after LeBail refinement using SXRPD pattern of $\mathrm{Ni}_{50} \mathrm{Mn}_{34} \mathrm{In}_{15.2} \mathrm{Al}_{0.8}$ MSMA for the (a) cubic austenite phase at $400 \mathrm{~K}$ and (b) $3 M$ modulated premartensite (PM) phase at $100 \mathrm{~K}$ in the $F m \overline{3} m$ and $P 2 / m$ space groups, respectively. The vertical tick marks above the difference profile represent the Bragg peak positions in (a) and (b). The inset of (a) shows the presence of (111) and (200) Bragg reflections characteristic of the $L 2_{1}$ ordering in the cubic austenite phase. The inset of (b) shows an enlarged view of the fit around the most intense Bragg peak and satellite reflections (marked as "PM" with their indices) related to the $3 M$ modulated premartensite phase. The temperature dependence of the $\mathrm{dc}$ magnetization, measured on zero field cooled sample during warming cycle, is shown in (c) for different magnetic fields. The enlarged view of (c) around the FM $T_{C}$, shown in (d), reveals a skewed diffuse peak due to the PM transition. The variation of the PM transition temperature $\left(T_{\mathrm{PM}}\right)$ with magnetic field is shown in (e). An enlarged view of SXRPD pattern around the most intense Bragg peak collected at $294 \mathrm{~K}$ under zero field (black dots connected with continuous line) and an external magnetic field of $2500 \mathrm{Oe}$ (red squares connected with continuous line) is shown in (f). Note the disappearance of the satellite peaks related to the premartensite (PM) phase under the magnetic field.

domain size of the PM phase keeps growing below $300 \mathrm{~K}$ after its nucleation around $T_{\mathrm{PM}}=300 \mathrm{~K}$. Moreover, the cubic (220) peak does not show any splitting down to the lowest temperature $100 \mathrm{~K}$ up to which we could collect the SXRPD patterns [see Fig. 3(e)]. We verified the absence of splitting in this peak even below $100 \mathrm{~K}$ using the laboratory source $\mathrm{X}$-ray powder diffraction data [see inset of Fig. 3(e)]. The absence of any splitting of the (220) cubic peak, shown in the inset of Fig. 3(e), down to $13 \mathrm{~K}$ is in marked contrast to that shown in Fig. S2(b) (see the Supplemental Material [57]) for $\mathrm{Ni}_{50} \mathrm{Mn}_{34} \mathrm{In}_{15.5} \mathrm{Al}_{0.5}$ alloy. This confirms the stability of the PM phase down to $13 \mathrm{~K}$ with preserved cubic symmetry without any discernible Bain distortion. The absence of any splitting of the (220) austenite peak, the appearance of the satellite peaks at $T \leqslant T_{\mathrm{PM}} \sim 300 \mathrm{~K}$ and the absence of any peak in the $\chi^{\prime}(T)$ plot in Fig. 1(b) below the $T_{\mathrm{PM}}$ corresponding to the martensite transition clearly confirms the suppression of the martensite phase in $\mathrm{Ni}_{50} \mathrm{Mn}_{34} \mathrm{In}_{15.2} \mathrm{Al}_{0.8}$ MSMA and the stabilization of the PM phase in the entire temperature range below $T_{\mathrm{PM}}$. These qualitative observations were verified by LeBail refinement as discussed below.
The LeBail refinement using the SXRPD pattern at $400 \mathrm{~K}$ [Fig. 4(a)] for the $\mathrm{Ni}_{50} \mathrm{Mn}_{34} \mathrm{In}_{15.2} \mathrm{Al}_{0.8}$ alloy for the cubic austenite phase in the space group $F m \overline{3} m$ confirmed that all the peaks in the SXRPD pattern could be indexed very well. The results of this refinement are shown in Fig. 4(a) which reveals an excellent fit between the observed and calculated profiles for the refined unit cell parameter $a=6.0169$ (1) $\AA$. The presence of (111) and (200) Bragg peaks shown in the inset of Fig. 4(a) confirms the ordered $L 2_{1}$ cubic structure for the austenite phase [56]. Having confirmed the singlephase nature and $L 2_{1}$ ordering in the cubic austenite phase of $\mathrm{Ni}_{50} \mathrm{Mn}_{34} \mathrm{In}_{15.2} \mathrm{Al}_{0.8}$, LeBail refinement using the SXRPD pattern was carried out at $100 \mathrm{~K}$ (i.e., well below $T_{\mathrm{PM}}$ ) for the $3 M$ modulated monoclinic structure in the space group $P 2 / m$, similar to that for the $\mathrm{Ni}_{50} \mathrm{Mn}_{34} \mathrm{In}_{15.5} \mathrm{Al}_{0.5}$ composition. The observed, calculated, and difference profiles so obtained, shown in Fig. 4(b), reveal excellent fit for the $3 M$ modulated monoclinic structure. The inset of Fig. 4(b) depicts an enlarged view of the LeBail fit around the most intense Bragg peak. The satellite peaks corresponding to the PM phase are marked as 'PM' along with their indices in this inset. The refined lattice parameters obtained after refinements are 
(a)

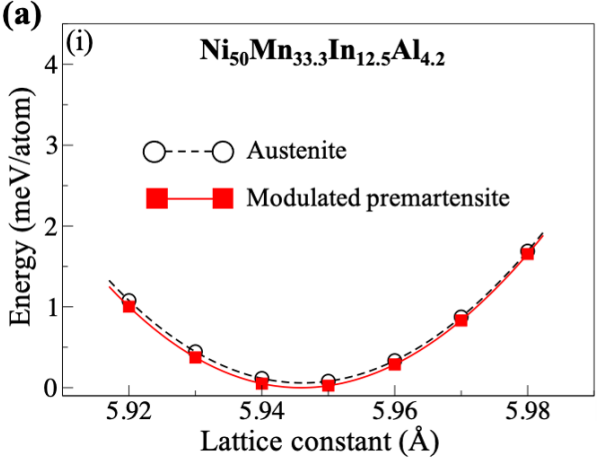

(b)

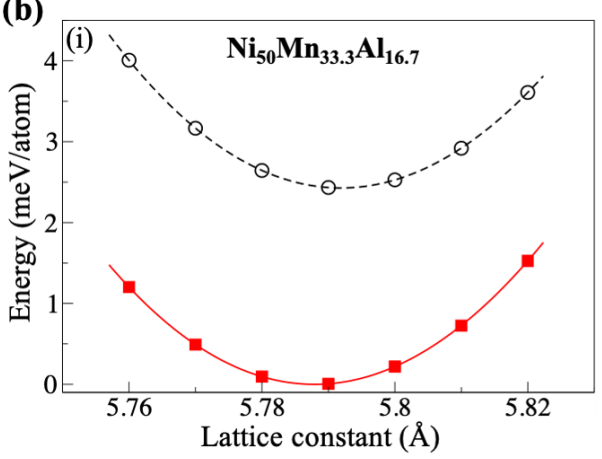

(ii)

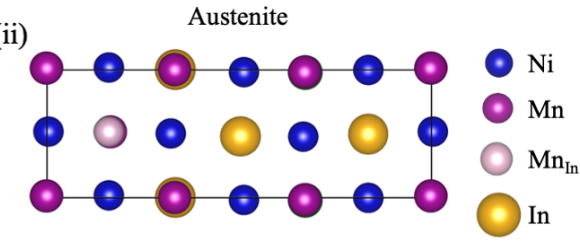

(iii)

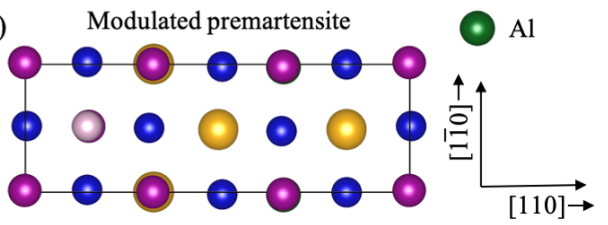

(ii)

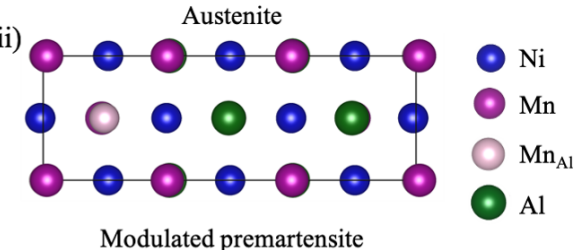

(iii)

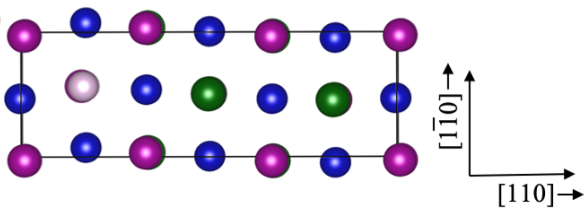

FIG. 5. Calculated total energies as a function of lattice constant in the austenite and modulated premartensite phases of (a) $\mathrm{Ni}_{50} \mathrm{Mn}_{33.3} \mathrm{In}_{12.5} \mathrm{Al}_{4.2}$ and (b) $\mathrm{Ni}_{50} \mathrm{Mn}_{33.3} \mathrm{Al}_{16.7}$. Supercells with relaxed atomic positions for the austenite and the modulated premartensite phases of $\mathrm{Ni}_{50} \mathrm{Mn}_{33.3} \mathrm{In}_{12.5} \mathrm{Al}_{4.2}$ and $\mathrm{Ni}_{50} \mathrm{Mn}_{33.3} \mathrm{Al}_{16.7}$ are shown in insets (ii), (iii) of (a) and (b), respectively. $\mathrm{Mn}_{\text {In }}\left(\mathrm{Mn}_{\mathrm{Al}}\right)$ indicate excess $\mathrm{Mn}$ atoms (i.e., above $25 \% \mathrm{Mn}$ content) in our simulation cells occupying In (Al) sublattice. The $\mathrm{Al}$ atoms are behind the In atoms in the insets (ii) and (iii) of (a).

$a=4.3823(2) \AA, b=5.6480(4) \AA, c=12.9754(4) \AA, \beta=$ $93.755(3)^{\circ}$ for the PM structure of $\mathrm{Ni}_{50} \mathrm{Mn}_{34} \mathrm{In}_{15.2} \mathrm{Al}_{0.8}$ at $100 \mathrm{~K}$. These parameters correspond to the $3 M$ modulated monoclinic structure as per the convention used in the literature for the modulated structures in MSMAs [21,76-80].

The magnetoelastic coupling has been suggested as one of the factors for the stabilization of the PM phase in MSMAs $[18,23,50,81]$. The effect of magnetoelastic coupling is manifested through the variation of $T_{\mathrm{PM}}$ with magnetic field $[18,19,23,50]$. Therefore, to investigate the possibility of magnetoelastic coupling in $\mathrm{Ni}_{50} \mathrm{Mn}_{34} \mathrm{In}_{15.2} \mathrm{Al}_{0.8}$ MSMA, we investigated the temperature dependence of the dc magnetization as a function of temperature $[M(T)]$ at different magnetic fields $(100,200,500,800$, and $1500 \mathrm{Oe})$ during the warming cycle on a zero field cooled sample (ZFCW protocol) shown in Fig. 4(c). The enlarged view of Fig. 4(c) around the diffuse peak in $M(T)$ is shown in Fig. 4(d) where the peak temperatures are marked by arrows. The variation of the peak temperatures in $M(T)$ with field, shown in Fig. 4(e), reveals that $T_{\mathrm{PM}}$ shifts towards the lower temperature side linearly with increasing magnetic field. This has been attributed to magnetoelastic coupling $[18,19,23,50]$. We verified the magnetoelastic coupling in $\mathrm{Ni}_{50} \mathrm{Mn}_{34} \mathrm{In}_{15.2} \mathrm{Al}_{0.8}$ further by recording high-resolution SXRPD patterns without and in the presence of magnetic field ( 0 and $2500 \mathrm{Oe}$ ) at $294 \mathrm{~K}$. The results of such measurements are shown in Fig. 4(f) around the most intense Bragg peak while the SXRPD pattern in full $2 \theta$ range is shown in Fig. S4 (see the Supplemental Material [57]). It is evident from Fig. 4(f) that the satellite peaks related to the PM phase (marked as "PM" for the zero field pattern) disappear completely at 2500 Oe. This confirms that the lattice and spin degrees of freedom are not only coupled but also play a crucial role on the stability of the PM phase of $\mathrm{Ni}_{50} \mathrm{Mn}_{34} \mathrm{In}_{15.2} \mathrm{Al}_{0.8}$ MSMA. It is worth mentioning here that the satellite peaks related to the PM phase are better resolved in Fig. 4(f) than Fig. 3(b) due to better peak to peak resolution for the lower energy $(25 \mathrm{keV})$ beam used for the former in contrast to $60 \mathrm{keV}$ beam used for the latter.

In order to obtain microscopic insight into the role of Al substitution on the stability of the PM phase of $\mathrm{Ni}$ Mn-In MSMA, we calculated total energies of both PM and austenite phases using first-principles simulations for three off-stoichiometric compositions, i.e., $\mathrm{Ni}_{50} \mathrm{Mn}_{33.3} \mathrm{In}_{16.7}$, $\mathrm{Ni}_{50} \mathrm{Mn}_{33.3} \mathrm{In}_{12.5} \mathrm{Al}_{4.2}$, and $\mathrm{Ni}_{50} \mathrm{Mn}_{33.3} \mathrm{Al}_{16.7}$ and the results so obtained are presented in Fig. 5. Excess Mn atoms (i.e., above 25\% Mn content) in our simulation cells [shown in insets (ii), (iii) of Figs. 5(a) and 5(b)] occupy the In/Al sublattice and couple ferromagnetically to the host $\mathrm{Mn}$ atoms in the two In containing alloys. The interaction between host and excess $\mathrm{Mn}$ atoms is, however, of antiferromagnetic nature in $\mathrm{Ni}_{50} \mathrm{Mn}_{33.3} \mathrm{Al}_{16.7}$. We have considered different chemical arrangements of excess $\mathrm{Mn}$, In, and $\mathrm{Al}$ on the In/Al sublattice in the present study and selected the atomic configuration with the lowest total energy for both the PM and the austenite phases. Our calculations indicate that the modulated PM phase is not stable for the Al-free $\mathrm{Ni}_{50} \mathrm{Mn}_{33.3} \mathrm{In}_{16.7}$ MSMA (results not shown here). During the process of ionic relaxation, the initially chosen atomic 
modulation for the modulated PM phase, as discussed in Ref. [82], disappears and the structure becomes identical to that of the nonmodulated austenite phase. We repeated the procedure several times with different values of amplitude of atomic modulation but always obtained the nonmodulated austenite phase after performing ionic relaxation. The Al substitution in place of In tends to stabilize the modulated PM phase as shown in the inset (i) of Fig. 5(a). For the $\mathrm{Ni}_{50} \mathrm{Mn}_{33.3} \mathrm{In}_{12.5} \mathrm{Al}_{4.2}$ MSMA, the total energy at $0 \mathrm{~K}$ for the PM phase around its equilibrium volume is slightly lower (approximately $0.1 \mathrm{meV} /$ atom) than that of the austenite phase. This suggests that $\mathrm{Al}$ substitution at the In site facilitates the stabilization of the modulated PM phase in $\mathrm{Ni}_{50} \mathrm{Mn}_{33.3} \mathrm{In}_{12.5} \mathrm{Al}_{4.2}$, albeit with tiny amplitudes of atomic modulations [inset (iii) of Fig. 5(a)] which nevertheless accounts for the small energy difference between the austenite and the PM phases for this alloy composition. In order to unambiguously establish the role of $\mathrm{Al}$ in stabilizing the modulated PM phase, we calculated the total energies of both the phases for an alloy with only $\mathrm{Al}$ in place of In, i.e., $\mathrm{Ni}_{50} \mathrm{Mn}_{33.3} \mathrm{Al}_{16.7}$. Inset (i) of Fig. 5(b) shows that the total energy at $0 \mathrm{~K}$ for the PM phase around its equilibrium volume is lower than that of the austenite phase by more than $2 \mathrm{meV} / \mathrm{atom}$ for this In free alloy. The amplitudes of the atomic modulations in the PM phase are also substantially larger in this case, as shown in inset (iii) of Fig. 5(b). All these suggest that the stability of the modulated PM phase increases with increasing Al content in place of In in Ni-Mn-In based MSMAs. It is important to mention that the temperature-dependent excitations, e.g., lattice vibrations, magnetic excitations, etc., are likely to alter the relative stability of the austenite phase with respect to that of the PM phase at higher temperatures [83]. Nevertheless, at low temperatures, our calculations predict PM phase to be more stable than the austenite phase for Al- substituted Ni-Mn-In based MSMAs.

Moreover, we note that the stability of the PM phase in $\mathrm{Ni}_{50} \mathrm{Mn}_{33.3} \mathrm{Al}_{16.7}$ is strongly dependent on the abovementioned antiferromagnetic interaction between host and the excess $\mathrm{Mn}$ atoms. For an artificially chosen ferromagnetic state (Fig. S7; see the Supplemental Material [57]) with all Mn spins aligned in the same direction, atomic modulations in the PM phase almost disappear resulting in negligible energy difference between the PM and the austenite phase. It may therefore be speculated that the origin of the missing PM phase in $\mathrm{Ni}_{50} \mathrm{Mn}_{33.3} \mathrm{In}_{16.7}$ lies in the ferromagnetic interaction between the host and the excess Mn atoms in this alloy. The substitution of $\mathrm{Al}$ in place of $\mathrm{In}$ in $\mathrm{Ni}_{50} \mathrm{Mn}_{33.3} \mathrm{In}_{16.7}$, however, tends to change the nature of this magnetic interaction from ferromagnetic to antiferromagnetic, which in turn facilitates the stabilization of the PM phase. Our calculations, thus, indicate a strong influence of magnetism on the stability of the
PM phase which corroborates the experimental finding for the absence of the PM phase in the presence of a modest external magnetic field [Fig. 4(f)].

\section{CONCLUSIONS}

To conclude, we presented here evidence for chemical pressure induced suppression of the main martensite transition and stabilization of the PM phase over a very wide temperature range from 300 to $\sim 5 \mathrm{~K}$ in a magnetic shape memory alloy $\mathrm{Ni}_{50} \mathrm{Mn}_{34} \mathrm{In}_{16}$ using magnetic susceptibility, synchrotron $\mathrm{x}$-ray powder diffraction studies, and first-principles calculations. The ac-susceptibility studies show that the stability of the martensite phase is suppressed while that of the premartensite phase enhanced with increasing $\mathrm{Al}$ content in place of In in $\mathrm{Ni}_{50} \mathrm{Mn}_{34} \mathrm{In}_{16}$. The temperature evolution of the SXRPD patterns provides robust evidence for the stabilization of the PM phase in $\mathrm{Ni}_{50} \mathrm{Mn}_{34} \mathrm{In}_{15.2} \mathrm{Al}_{0.8}$ MSMA. We have also shown that the $T_{\mathrm{PM}}$ decreases with increasing magnetic field indicating the role of magnetoelastic coupling. The disappearance of the satellite peaks of the PM phase in the SXRPD pattern in the presence of an external magnetic field provides direct evidence for the crucial role of magnetoelastic coupling in the stabilization of the PM phase in the base and $\sim 5 \% \mathrm{Al}$ substituted alloy compositions. Our first-principles calculations not only corroborate the experimental findings but also provide microscopic insight into the role of $\mathrm{Al}$ substitution at the In site on the stabilization of PM phase in the ground state of Ni-Mn-In alloys. Our results, thus, puts forward Al-substituted Ni-Mn-In MSMA as an ideal system for investigating the physics of precursor phenomena in MSMAs.

\section{ACKNOWLEDGMENTS}

S.S. thanks UGC-DAE CSR, Indore for financial support through the "CRS" scheme. S.S. is thankful to the Science and Engineering Research Board of India for financial support through the award of a Ramanujan Fellowship (Grant No. SB/S2/RJN-015/2017) and an Early Career Research Award (Grant No. ECR/2017/003186). This work was partially carried out using the facilities of UGC-DAE CSR, Indore. Portions of this research were conducted at the light source PETRA III of DESY, a member of the Helmholtz Association. Financial support from the Department of Science and Technology, Government of India, within the framework of the India@DESY is gratefully acknowledged. We would like to thank the beamline scientists Dr. M. Etter and Dr. J. Tseng for their help in setting up the experiments. Authors thank G. Bais and M. Polentarutti of Elettra-Sincrotrone Trieste for their help in the setup for SXRPD measurements under magnetic field. K.K.D thanks DST for providing fellowship through DST- INSPIRE scheme.
[1] K. Otsuka and C. M. Wayman, Shape Memory Materials (Cambridge University Press, Cambridge, UK, 1999).

[2] K. Otsuka and X. Ren, Prog. Mater. Sci. 50, 511 (2005).

[3] S. M. Shapiro, Y. Noda, Y. Fujii, and Y. Yamada, Phys. Rev. B 30, 4314 (1984).
[4] J. M. Jani, M. Leary, A. Subic, and M. A. Gibson, Mater. Des. 56, 1078 (2014).

[5] J. Bowles and J. Mackenzie, Acta Metall. 2, 129 (1954).

[6] Z. Nishiyama, Martensite Transformation (Academic, New York, 1978). 
[7] K. Bhattacharya, Microstructure of Martensite: Why it Forms and How it Gives Rise to the Shape-Memory Effect (Oxford University Press, New York, 2003).

[8] J. A. Venables, Philos. Mag. 7, 35 (1962).

[9] G. D. Sandrock, A. J. Perkins, and R. F. Hehemann, Metall. Trans. 2, 2769 (1971).

[10] S. M. Shapiro, B. X. Yang, Y. Noda, L. E. Tanner, and D. Schryvers, Phys. Rev. B 44, 9301 (1991).

[11] Z. Xingzhong, W. Xingfang, and K. Tsun, Acta Metall. 37, 1783 (1989).

[12] I. Robertson and C. Wayman, Philos. Mag. A 48, 443 (1983).

[13] A. Nagasawa and Y. Morii, Mater. Trans. JIM 34, 855 (1993).

[14] A. Nagasawa, K. Kita, Y. Morii, K. Fuchizaki, S. Katano, S. Funahashi, and C. HR, Mater. Trans. JIM 32, 1011 (1991).

[15] A. Planes and L. Mañosa, in Solid State Physics (Elsevier, New York, 2001), Vol. 55, pp. 159-267.

[16] A. Zheludev, S. M. Shapiro, P. Wochner, and L. E. Tanner, Phys. Rev. B 54, 15045 (1996).

[17] J. Worgull, E. Petti, and J. Trivisonno, Phys. Rev. B 54, 15695 (1996).

[18] A. Planes, E. Obradó, A. Gonzalez-Comas, and L. Mañosa, Phys. Rev. Lett. 79, 3926 (1997).

[19] F. Zuo, X. Su, and K. Wu, Phys. Rev. B 58, 11127 (1998).

[20] T. Castán, E. Vives, and P.-A. Lindgård, Phys. Rev. B 60, 7071 (1999).

[21] P. J. Brown, J. Crangle, T. Kanomata, M. Matsumoto, K.-U. Neumann, B. Ouladdiaf, and K. R. A. Ziebeck, J. Phys.: Condens. Matter 14, 10159 (2002).

[22] S. Singh, J. Bednarcik, S. R. Barman, C. Felser, and D. Pandey, Phys. Rev. B 92, 054112 (2015).

[23] Y. Wu, S. Guo, S. Yu, H. Cheng, R. Wang, H. Xiao, L. Xu, R. Xiong, Y. Liu, Z. Xia, and C. Yang, Sci. Rep. 6, 26068 (2016).

[24] S. Zuo, F. Liang, Y. Zhang, L. Peng, J. Xiong, Y. Liu, R. Li, T. Zhao, J. Sun, and F. Hu, Phys. Rev. Mater. 2, 104408 (2018).

[25] K. Ullakko, J. Mater. Eng. Perform. 5, 405 (1996).

[26] A. Sozinov, A. Likhachev, N. Lanska, and K. Ullakko, Appl. Phys. Lett. 80, 1746 (2002).

[27] K. Ullakko, J. K. Huang, C. Kantner, R. C. O’Handley, and V. V. Kokorin, Appl. Phys. Lett. 69, 1966 (1996).

[28] S. J. Murray, M. Marioni, S. M. Allen, R. C. O'Handley, and T. A. Lograsso, Appl. Phys. Lett. 77, 886 (2000).

[29] F.-x. Hu, B.-g. Shen, J.-r. Sun, and G.-h. Wu, Phys. Rev. B 64, $132412(2001)$.

[30] X. Moya, E. Defay, V. Heine, and N. D. Mathur, Nat. Phys. 11, 202 (2015).

[31] T. Krenke, E. Duman, M. Acet, E. F. Wassermann, X. Moya, L. Mañosa, and A. Planes, Nat. Mater. 4, 450 (2005).

[32] T. Krenke, E. Duman, M. Acet, E. F. Wassermann, X. Moya, L. Mañosa, A. Planes, E. Suard, and B. Ouladdiaf, Phys. Rev. B 75, 104414 (2007).

[33] T. Gottschall, E. Stern-Taulats, L. Mañosa, A. Planes, K. P. Skokov, and O. Gutfleisch, Appl. Phys. Lett. 110, 223904 (2017).

[34] C. Biswas, R. Rawat, and S. Barman, Appl. Phys. Lett. 86, 202508 (2005).

[35] S. Banik, S. Singh, R. Rawat, P. Mukhopadhyay, B. Ahuja, A. Awasthi, S. Barman, and E. Sampathkumaran, J. Appl. Phys. 106, 103919 (2009)

[36] S. Yu, Z. Liu, G. Liu, J. Chen, Z. Cao, G. Wu, B. Zhang, and X. Zhang, Appl. Phys. Lett. 89, 162503 (2006).
[37] T. Chabri, A. Awasthi, K. Ghosh, A. Venimadhav, and T. Nath, Mater. Res. Express 5, 086511 (2018).

[38] Y. Kuo, K. Sivakumar, H. Chen, J. Su, and C.-S. Lue, Phys. Rev. B 72, 054116 (2005).

[39] K. Priolkar, P. Bhobe, S. D. Sapeco, and R. Paudel, Phys. Rev. B 70, 132408 (2004).

[40] Z. Han, B. Qian, D. Wang, P. Zhang, X. Jiang, C. Zhang, and Y. Du, Appl. Phys. Lett. 103, 172403 (2013).

[41] Z. Li, C. Jing, J. Chen, S. Yuan, S. Cao, and J. Zhang, Appl. Phys. Lett. 91, 112505 (2007).

[42] S. Chatterjee, S. Giri, S. De, and S. Majumdar, Phys. Rev. B 79 , 092410 (2009).

[43] I. Dubenko, A. K. Pathak, S. Stadler, N. Ali, Y. Kovarskii, V. N. Prudnikov, N. S. Perov, and A. B. Granovsky, Phys. Rev. B 80 , 092408 (2009).

[44] A. De, A. K. Singh, S. Singh, and S. Nair, Phys. Rev. B 103, L020404 (2021).

[45] R. Nevgi and K. Priolkar, Appl. Phys. Lett. 112, 022409 (2018).

[46] S. Singh, J. Nayak, A. Rai, P. Rajput, A. H. Hill, S. R. Barman, and D. Pandey, J. Phys.: Condens. Matter 25, 212203 (2013).

[47] V. V. Khovailo, T. Takagi, A. D. Bozhko, M. Matsumoto, J. Tani, and V. G. Shavrov, J. Phys.: Condens. Matter 13, 9655 (2001).

[48] J. Enkovaara, A. Ayuela, L. Nordström, and R. M. Nieminen, Phys. Rev. B 65, 134422 (2002).

[49] H. Cheng, Z. C. Xia, R. L. Wang, M. Wei, Z. Jin, S. Huang, C. Shang, H. Wu, X. X. Zhang, G. L. Xiao, and Z. W. Ouyang, J. Phys. D: Appl. Phys. 50, 135004 (2017).

[50] S. Ma, H. Xuan, C. Zhang, L. Wang, Q. Cao, D. Wang, and Y. Du, Appl. Phys. Lett. 97, 052506 (2010).

[51] S. Ma, D. Wang, Z. Zhong, J. Luo, J. Xu, and Y. Du, Appl. Phys. Lett. 102, 032407 (2013).

[52] Y. T. Cui, J. L. Chen, G. D. Liu, G. H. Wu, and W. L. Wang, J. Phys.: Condens. Matter 16, 3061 (2004).

[53] H. Chou and S. M. Shapiro, Phys. Rev. B 48, 16088 (1993).

[54] R. Ranjan, S. Banik, S. R. Barman, U. Kumar, P. K Mukhopadhyay, and D. Pandey, Phys. Rev. B 74, 224443 (2006).

[55] S. Singh, V. Petricek, P. Rajput, A. H. Hill, E. Suard, S. R. Barman, and D. Pandey, Phys. Rev. B 90, 014109 (2014).

[56] P. Devi, S. Singh, B. Dutta, K. Manna, S. D’Souza, Y. Ikeda, E. Suard, V. Petricek, P. Simon, and P. Werner, Phys. Rev. B 97, 224102 (2018).

[57] See Supplemental Material at http://link.aps.org/supplemental/ 10.1103/PhysRevMaterials.5.113607 for experimental details, computational methods, measurements (SXRPD, lab-XRD, magnetization, resistivity), and LeBail refinements. Also see Refs. [58-68].

[58] B. Holt, J. Diaz, J. Huber, and C. A. Luengo, Rev. Bras. Fis. 8, 155 (1978).

[59] S. Singh, P. Kushwaha, F. Scheibel, H.-P. Liermann, S. Barman, M. Acet, C. Felser, and D. Pandey, Phys. Rev. B 92, 020105 (2015).

[60] S. Singh, M. Maniraj, S. W. D’Souza, R. Ranjan, and S. R. Barman, Appl. Phys. Lett. 96, 081904 (2010).

[61] P. Lotti, S. Milani, M. Merlini, B. Joseph, F. Alabarse, and A. Lausi, J. Synchrotron Radiat. 27, 222 (2020).

[62] G. Kresse and J. Hafner, Phys. Rev. B 47, 558 (1993).

[63] G. Kresse and J. Furthmüller, Comput. Mater. Sci. 6, 15 (1996). 
[64] G. Kresse and J. Furthmüller, Phys. Rev. B 54, 11169 (1996).

[65] P. E. Blöchl, Phys. Rev. B 50, 17953 (1994).

[66] J. P. Perdew, K. Burke, and M. Ernzerhof, Phys. Rev. Lett. 77, 3865 (1996).

[67] H. J. Monkhorst and J. D. Pack, Phys. Rev. B 13, 5188 (1976).

[68] J. Rodrigues-Carvajal, FULLPROF, a Rietveld refinement and pattern matching analysis program version 2016, Laboratoire Leon Brillouin, CEA-CNRS, France http://www.ill.eu/sites/ fullprof/.

[69] K. Priolkar, P. Bhobe, D. Lobo, S. D’Souza, S. Barman, A. Chakrabarti, and S. Emura, Phys. Rev. B 87, 144412 (2013).

[70] T. Krenke, M. Acet, E. F. Wassermann, X. Moya, L. Mañosa, and A. Planes, Phys. Rev. B 73, 174413 (2006).

[71] M. Siewert, M. Gruner, A. Dannenberg, A. Chakrabarti, H. Herper, M. Wuttig, S. Barman, S. Singh, A. Al-Zubi, and T. Hickel, Appl. Phys. Lett. 99, 191904 (2011).

[72] P. Entel, M. Siewert, M. E. Gruner, A. Chakrabarti, S. R. Barman, V. V. Sokolovskiy, and V. D. Buchelnikov, J. Alloys Compd. 577, S107 (2013).

[73] S. Banik, A. Chakrabarti, U. Kumar, P. Mukhopadhyay, A. Awasthi, R. Ranjan, J. Schneider, B. Ahuja, and S. Barman, Phys. Rev. B 74, 085110 (2006).
[74] L. Manosa, D. Gonzalez-Alonso, A. Planes, E. Bonnot, M. Barrio, J.-L. Tamarit, S. Aksoy, and M. Acet, Nat. Mater. 9, 478 (2010).

[75] S. Singh, B. Dutta, S. W. D’Souza, M. Zavareh, P. Devi, A. Gibbs, T. Hickel, S. Chadov, C. Felser, and D. Pandey, Nat. Commun. 8, 1006 (2017).

[76] S. Singh, S. D’Souza, J. Nayak, L. Caron, E. Suard, S. Chadov, and C. Felser, Phys. Rev. B 93, 134102 (2016).

[77] S. Singh, R. Rawat, and S. R. Barman, Appl. Phys. Lett. 99, 021902 (2011).

[78] S. Singh, K. R. A. Ziebeck, E. Suard, P. Rajput, S. Bhardwaj, A. M. Awasthi, and S. R. Barman, Appl. Phys. Lett. 101, 171904 (2012).

[79] L. Righi, F. Albertini, G. Calestani, L. Pareti, A. Paoluzi, C. Ritter, P. A. Algarabel, L. Morellon, and M. Ricardo Ibarra, J. Solid State Chem. 179, 3525 (2006).

[80] S. Singh, S. R. Barman, and D. Pandey, Z. Kristallogr. 230, 13 (2015).

[81] A. K. Singh, S. Singh, and D. Pandey, Phys. Rev. B 104, 064110 (2021).

[82] B. Dutta, A. Çakır, C. Giacobbe, A. Al-Zubi, T. Hickel, M. Acet, and J. Neugebauer, Phys. Rev. Lett. 116, 025503 (2016).

[83] M. A. Uijttewaal, T. Hickel, J. Neugebauer, M. E. Gruner, and P. Entel, Phys. Rev. Lett. 102, 035702 (2009). 